Rev. Int. Contam. Ambie. 35 (Residuos solidos en México) 19-27, 2019

DOI: 10.20937/RICA.2019.35.esp02.03

\title{
EVALUACIÓN DE LOS SISTEMAS DE MANEJO DE RESIDUOS EN CUATRO MUNICIPIOS DE JALISCO, MÉXICO
}

Evaluation of waste management systems in four municipalities of Jalisco, Mexico

\author{
Gerardo BERNACHE PÉREZ
}

Centro de Investigaciones y Estudios Superiores en Antropología Social (CIESAS), Unidad Occidente. Av. España 1359, Col. Moderna, 44190 Guadalajara, Jalisco, México

Correo electrónico: gerardo.bernache@gmail.com

(Recibido agosto 2018; aceptado agosto 2018)

Palabras clave: disposición final, Guadalajara, residuos sólidos urbanos, valorización de residuos

\section{RESUMEN}

En México, las políticas públicas y el marco regulatorio sobre los residuos sólidos urbanos (RSU) han avanzado en las últimas dos décadas, de tal manera que se ha pasado de los sistemas de limpia y manejo de la basura, a los sistemas de gestión integral de los RSU, donde la prioridad es el aprovechamiento de los materiales. Ya no es pertinente hablar de basura, sino de residuos y materiales que pueden ser reciclados, convertidos en composta y valorizados de múltiples formas. Sin embargo, las propuestas de políticas y programas de gestión no se han resultado en cambios sustanciales en las formas, técnicas, infraestructura y sistemas de manejo de los residuos. En teoría se ha avanzado, pero en la práctica no. Los residuos siguen siendo manejados como basura, es decir, como desperdicios no útiles que tienen como destino principal el entierro en vertederos poco seguros desde la perspectiva ambiental. En este artículo se comparan datos de producción de RSU y los montos de residuos inorgánicos que se separan para el reciclaje, los montos del componente orgánico que se van a composteras o son valorizados en la producción y destrucción controlada de metano. Se comparan datos de 1997 y 2017 para cuatro municipalidades. Se puede ver un avance relativo, de un 5 a $8 \%$ de aprovechamiento del total de residuos, pero éste es un avance mínimo en relación con el problema de generación de RSU en una zona metropolitana como Guadalajara, la segunda urbe más poblada de la República mexicana.

Key words: final disposition, Guadalajara, urban solid waste, waste valorization

\begin{abstract}
In Mexico, public policies and the regulatory framework on urban solid waste (USW) have advanced notably during the last two decades, moving from old sanitation systems of garbage collection and disposal, to integrated systems of waste management, where the priority is the valorization of materials. It is no longer relevant to focus on garbage, but to promote recycling of waste materials and composting organic wastes, as well as adding value to waste materials using innovative process. However, new proposals for integrated waste management programs have not resulted in substantial changes
\end{abstract}


at municipal level. In theory, some progress is evident, but in practice the advance is minor. Solid wastes are still managed as garbage; as non-useful waste whose main destination is a local landfill. This article compares USW production data with the amounts of inorganic materials separated for recycling and the amounts of the organic component that go to compost or are valued in other ways. Data from 1997 and 2017 for four municipalities are compared. The results show some progress in valorization, increasing from 5 to $8 \%$ of total USW, but this is a minor advance in relation to the large problem of generation of USW in a metropolitan area such as Guadalajara, the second most populated city in Mexico.

\section{INTRODUCCIÓN}

Los marcos normativos federal y estatal, los programas y políticas públicas, y los discursos en relación con el manejo de los RSU en México, han evolucionado sustancialmente en las últimas dos décadas; sin embargo, tanto las prácticas de consumo y disposición de RSU como las estrategias de manejo no siempre han evolucionado al mismo ritmo para estar a la altura de los ideales propuestos.

Los residuos sólidos urbanos son el objeto de esta investigación. La Ley General para la Protección y Gestión Integral de Residuos Sólidos ha definido este tipo de residuos en su artículo 5, inciso XXXIII:

XXXIII. Residuos sólidos urbanos: los generados en las casas habitación, que resultan de la eliminación de los materiales que utilizan en sus actividades domésticas, de los productos que consumen y de sus envases, embalajes o empaques; los residuos que provienen de cualquier otra actividad dentro de establecimientos o en la vía pública que genere residuos con características domiciliarias, y los resultantes de la limpieza de las vías y lugares públicos, siempre que no sean considerados por esta Ley como residuos de otra índole (SEMARNAT 2003).

Es decir, la ley se enfoca a los RSU que ingresan a los sistemas de recolección establecidos por los ayuntamientos. Esto es importante, ya que no todos los RSU ingresan a los sistemas de recolección. Por ejemplo, es común que empresas e instituciones que producen grandes cantidades de un producto o material lo separen y comercialicen. Asimismo, en la actualidad hay un gran número de pepenadores que revisan en las calles las bolsas o botes de residuos que desechan los usuarios y rescatan materiales útiles con valor comercial antes de que los levante el camión recolector.
Este artículo se propone estudiar y analizar las prácticas recientes en el manejo de RSU en los cuatro municipios centrales de la Zona Metropolitana de Guadalajara (ZMG): Guadalajara, Zapopan, Tlaquepaque y Tonalá. Este acotamiento se debe a que no se cuenta con datos sistemáticos sobre generación y manejo de residuos correspondientes a la década de 1990 para otros municipios que se han incorporado más recientemente a la zona metropolitana (Tlajomulco, El Salto, Juanacatlán e Ixtlahuacán de los Membrillos).

En 2015 la zona conurbada de la capital de Jalisco contaba con 7.8 millones de habitantes (INEGI 2015, IIEG 2017), con un $87 \%$ de población urbana y un promedio de escolaridad de 9.2 (secundaria terminada), siendo el comercio la principal actividad económica. La población total de los cuatro municipios considerados en este estudio era de poco más de cuatro millones de habitantes. El cuadro I muestra la población de cada municipalidad.

CUADRO I. POBLACIÓN DE LOS MUNICIPIOS ESTUDIADOS EN 2017

\begin{tabular}{lc}
\hline Municipios & Total de habitantes \\
\hline Guadalajara & 1448467 \\
Zapopan & 1372240 \\
Tlaquepaque & 689432 \\
Tonalá & 568278 \\
Total & 4078417 \\
\hline
\end{tabular}

Fuente: estimaciones propias con base en datos del INEGI (2015) y el IIEG (2017)

En cuanto al marco normativo que establece los criterios para el manejo de residuos, se pueden mencionar los principales instrumentos correspondientes, empezando en el nivel federal con la Ley General para la Prevención y Gestión Integral de Residuos (LGPGIR) y su reglamento (SEMARNAT 2003, 
2006). En la LGPGIR se establecen las directrices generales para el manejo idóneo de residuos. En Jalisco también existe un marco regulatorio : la Ley de Gestión Integral de Residuos del Estado de Jalisco, publicado por el gobierno estatal en 2007 y su reglamento respectivo, publicado en 2011 (SEMADES 2007, 2011). Entonces, tanto a nivel federal como estatal se marca la pauta para transitar del manejo de basura a la gestión integral de RSU. Se propone dejar atrás el antiguo proceso de limpia, donde la autoridad realiza una actividad mecánica de recolección, transporte y entierro de los desechos para dar paso a un nuevo proceso que considera importante la participación ciudadana, empezando por un consumo responsable que lleva a la minimización de la producción de RSU; luego promueve las prácticas de separación de residuos en la fuente generadora para dar lugar a valorización de residuos, lo cual generan composta, captura de biogás y reciclaje de materiales inorgánicos. Todo esto involucra a la ciudadanía, a partir de la promoción de una nueva cultura con la educación ambiental y el impulso al consumo verde. Las políticas públicas y el marco normativo en materia de residuos tienen como objetivo central reducir la producción de RSU, incrementar la separación para aprovechar los materiales y de esta forma propiciar una baja significativa de los montos de residuos que se entierran en sitios de disposición final.

El avance en las propuestas para el manejo sustentable de los RSU se debe reflejar en cambios significativos en los indicadores básicos de su producción y gestión, en este caso en la zona metropolitana de Guadalajara:

1. Montos de RSU generados por día. Este indicador presenta el monto total de RSU que ingresan en el sistema de recolección municipal, expresado en toneladas por día. Es un indicador global en un municipio.

2. Producción diaria de RSU per cápita. Se refiere al monto de RSU que le corresponde a cada habitante de un municipio. Es una suma de los desechos domésticos y los desechos municipales, es decir, de lo que cada persona produce de residuos en forma directa, más el monto que le corresponde de los residuos de otras fuentes municipales, espacios $\mathrm{y}$ vías públicas, mercados y tianguis, parques y jardines, instituciones y más.

3. Separación de residuos inorgánicos para su aprovechamiento. Refleja la manera en que se manejan los residuos inorgánicos y los materiales susceptibles de valorización y reciclaje, como plásticos, vidrio, metal, papel, cartón y otros. Se refiere a montos y porcentajes de separación para el reciclaje, principalmente.

4. Valorización del componente orgánico de los residuos. Se centra en la forma en que se manejan los residuos orgánicos, principalmente desechos y desperdicios de alimentos, así como los residuos de plantas y jardinería. Su principal forma de valorización es la producción de composta.

5. Montos de RSU enterrados en sitios de disposición final. Indica el porcentaje y los montos de RSU recolectados en las municipalidades que terminan por ser enterrados en sitios de disposición final (vertederos o rellenos sanitarios).

Estos cinco indicadores ofrecen información básica relevante sobre el crecimiento de la producción de RSU y la forma en que dichos residuos se manejan a nivel municipal, su aprovechamiento y el impacto ambiental. Nos brindan un panorama general de la gestión de residuos y su avance en la dirección de la gestión integral, de acuerdo con lo establecido en la LGPGIR (SEMARNAT 2003).

Este artículo presenta datos para cada uno de los cinco indicadores en dos momentos: 1997 y 2017. Durante este periodo de 20 años se puede observar el avance en los planteamientos, las políticas públicas y los programas dedicados a impulsar la gestión integral de RSU; sin embargo, los datos de producción y valorización de residuos indicarán si se ha generado un cambio en los procesos de gestión de RSU que realizan las autoridades municipales.

\section{MATERIALES Y METODOS}

Los datos de 1997 fueron publicados por Bernache et al. (1998) como resultado de un estudio detallado sobre la producción y gestión de RSU en los cuatro municipios identificados. En ese estudio se realizó la caracterización de residuos a partir de una muestra de 300 hogares en la ZMG, además de obtener registros de la producción diaria de residuos de los cuatro ayuntamientos y las bitácoras de ingreso a los sitios de disposición final que operaban en ese entonces.

La investigación de campo se realizó en 2015, y en este artículo se hizo una estimación de datos con el fin de presentar cifras actualizadas a 2017. Para la investigación se utilizó una estrategia metodológica de entrevistas a los funcionarios responsables de las dependencias de aseo público y de medio ambiente de los cuatro municipios. En total se realizaron cinco entrevistas en las que se utilizó una guía con un 
listado de temas y preguntas relacionadas con las diferentes etapas de la producción, el manejo y la gestión integral de RSU. Además, al finalizar las entrevistas se solicitaron datos sobre las cifras de generación de residuos, los sistemas de recolección, la transferencia, programas de separación y de participación ciudadana, tratamiento y valorización de residuos, y disposición final.

En cuanto a los recorridos de observación, éstos se llevaron a cabo con la lógica de la observación etnográfica en algunos puntos clave como las estaciones de transferencia Los Belenes, en Zapopan, y Coyula, en Tonalá; y los rellenos sanitarios Picachos, en Zapopan, y Los Laureles, en Tonalá. Se redactaron notas de campo y también se hicieron anotaciones en una lista con elementos a observar en los recorridos. En general se observaron las prácticas, el orden y la organización, el control de los procesos, los puntos débiles en el manejo, impactos ambientales en general y la incidencia de degradación ambiental.

Por último, se realizó una búsqueda en Internet y se incorporaron datos publicados recientemente por autores que han estudiado la producción y el manejo de residuos en el área metropolitana de Guadalajara (Aguayo 2013, Vilches 2013) y que colaboraron con el autor.

\section{RESULTADOS}

Los resultados se presentan en cinco apartados que corresponden a cada uno de los indicadores de producción de residuos total, generación per cápita, separación y valorización, y disposición final en vertederos.

\section{Montos de RSU}

Los cuatro municipios en estudio generaban 3119 t de RSU en 1997, mientras que en 2017 la generación alcanzó 4927 t/día (Bernache et al. 1998, Bernache 2013, 2015). El aumento en la generación de residuos se relaciona con tres factores principales: crecimiento de la población metropolitana, con 653533 habitantes más (INEGI 2015, IIEG 2017); incremento en las actividades económicas de producción y consumo urbano en la ZMG; y aumento en la producción de residuos por parte de los individuos y las familias.

En 2017 se produjeron 1807 t más de RSU que en 1997, un incremento del $58 \%$ en los montos totales para la metrópoli, mientras que la población residente en la ciudad creció tan solo un $19 \%$. El cuadro II presenta los datos para cada municipio.

El principal crecimiento en la producción de RSU se observa en los municipios de Tlaquepaque y Tonalá, con $127 \%$ y $348 \%$, respectivamente. E1 municipio de Guadalajara tuvo un decremento de $10 \%$ en población, pero aun así su producción de residuos creció en $16 \%$.

\section{Producción per cápita}

El cuadro III muestra las cifras correspondientes a producción per cápita de RSU en 1997 y 2017. Esta cifra es un promedio de la relación entre el total de RSU dividido entre el número de habitantes de la zona. En los 18 años del periodo de estudio, la producción creció en $297 \mathrm{~g}$, un aumento de $32.6 \%$ en el que inciden solamente dos factores: el aumento en la producción de residuos por persona y el aumento derivado del crecimiento en las actividades económicas de producción y consumo.

\section{Separación de materiales de residuos inorgánicos}

Se identifica a los residuos inorgánicos como aquellos materiales procesados como plásticos, papel, cartón, vidrio, metales y otros. Se contabiliza la separación a partir de los residuos que ingresan en el sistema de recolección municipal. Es importante señalar que a partir de 2016 el ayuntamiento de Guadalajara inició el Programa de Puntos Limpios, pero aún no se han reportado los montos de residuos orgánicos que se han captado.

CUADRO II. COMPARACIÓN DE MONTOS DE RESIDUOS GENERADOS

\begin{tabular}{lcccccc}
\hline \multirow{2}{*}{ Municipios } & \multicolumn{2}{c}{1997} & \multicolumn{2}{c}{2017} & $\begin{array}{c}\text { Diferencia } \\
\text { (\% de RSU) }\end{array}$ & $\begin{array}{c}\text { Diferencia } \\
\text { (\% de habitantes) }\end{array}$ \\
\cline { 2 - 5 } & Habitantes & Toneladas & Habitantes & Toneladas & & \\
\hline Guadalajara & 1612697 & 1506 & 1448467 & 1750 & 16 & -10 \\
Zapopan & 1009817 & 1093 & 1372240 & 1658 & 52 & 36 \\
Tlaquepaque & 491061 & 367 & 689432 & 833 & 127 & 40 \\
Tonalá & 311309 & 153 & 568278 & 687 & 348 & 83 \\
Total & 3424884 & 3119 & 4078417 & 4927 & 58 & 19 \\
\hline
\end{tabular}


CUADRO III. PRODUCCION DE RESIDUOS PER CÁPITA

\begin{tabular}{cc}
\hline Año & RSU per cápita* \\
\hline 1997 & 911 \\
2017 & 1208 \\
Diferencia & 297 \\
\hline
\end{tabular}

*En g/día

En 1997 se reportaba que el monto de residuos separados para su aprovechamiento por parte de asociaciones vecinales, iglesias, escuelas, trabajadores de recolección, pepenadores y otros grupos era de un $5 \%$ del total de los residuos (Bernache et al. 1998). Ya entonces se señalaba que no existían programas formales de separación impulsados desde los ayuntamientos:

En ninguno de los cuatro municipios de la ZMG existe un programa serio de manejo de los residuos que resulte en: 1) la reducción significativa e RSM que se generan diariamente; y 2) la separación de un porcentaje importante de los subproductos de los RSM para aprovecharlos como materia prima a comercializar para el reciclaje (Bernache et al. 1998).

En 2008 hubo un cambio significativo con la publicación de la norma de separación de residuos NAE-SEMADES-007/2008 (SEMADES 2008), que estableció la obligatoriedad de la separación primaria de residuos en Jalisco para los residentes y recolectores. Es decir, los usuarios debían entregar sus residuos separados en tres categorías: orgánicos, inorgánicos y sanitarios. A partir de la publicación de esta norma un sector muy importante de la población empezó a separar residuos en casa y entregarlos separados al sistema de recolección. Se establecieron días de la semana para la recolección de orgánicos y sanitarios, y otros para inorgánicos. Sin embargo, los sistemas municipales y las empresas concesionarias no pudieron mantener este sistema de recolección separada, y después de unos meses todos los residuos empezaron a recibirse mezclados.

De ahí que para 2017 los montos de separación de residuos habían crecido de forma modesta para ubicarse en un $8 \%$ de los RSU (Aguayo 2013, Vilches 2013), que actualmente corresponden a 394 t/día.

Los principales agentes que participan en la separación de residuos son cinco y se presentan en el cuadro IV, así como un estimado de su contribución porcentual al total de residuos separados. En este cuadro se puede apreciar que cuatro de los cinco agentes que participan en la separación de residuos son actores que desarrollan una actividad informal y que su motivación principal es completar sus ingresos o bien obtener una retribución por la venta de materiales que separan de la basura. Incluso el personal de recolección municipal realiza actividades de separación de forma paralela a su empleo. Además, en los camiones recolectores de Zapopan y Tlaquepaque se suman dos o tres voluntarios por unidad, los cuales obtienen una remuneración a partir de las propinas de los usuarios y la venta de materiales. En Jalisco se tienen registradas más de 300 empresas de recolección contratada de residuos. En su mayoría son micro, pequeñas y medianas empresas cuyos empleados también realizan actividades de separación y comercialización de materiales reciclables antes de llevar su carga de residuos al sitio de disposición final.

CUADRO IV. SEPARACIÓN DE RESIDUOS INORGÁNICOS

\begin{tabular}{|c|c|c|}
\hline $\begin{array}{l}\text { Tipos de } \\
\text { separación }\end{array}$ & Porcentaje & $\begin{array}{c}\text { Montos de materiales } \\
\text { separados* }\end{array}$ \\
\hline $\begin{array}{l}\text { Pepenadores en sitios de } \\
\text { disposición final y plantas } \\
\text { de transferencia }\end{array}$ & 60 & 236.5 \\
\hline $\begin{array}{l}\text { Personal de recolección } \\
\text { en los camiones }\end{array}$ & 10 & 39.4 \\
\hline Pepena en las calles & 12 & 47.3 \\
\hline $\begin{array}{l}\text { Organizaciones de } \\
\text { ciudadanos, escuelas } \\
\text { y universidades }\end{array}$ & 8 & 31.5 \\
\hline Recolectores privados & 10 & 39.4 \\
\hline Total & 100 & 394.1 \\
\hline
\end{tabular}

*En t/día

Fuente: datos del autor, Aguayo (2013) y Vilches (2013)

Solamente las organizaciones ciudadanas, escuelas, asociaciones de colonos y universidades tienen programas formales y organizados para la separación de residuos sólidos. Los ayuntamientos, que son los responsables directos del manejo de residuos sólidos, carecían hasta 2017 de programas relevantes de separación de residuos inorgánicos.

\section{Valorización de residuos orgánicos}

En el municipio de Zapopan hay dos programas de empresas privadas para la valorización del componente orgánico de los residuos.

Se estima que un $53 \%$ de los RSU son de tipo orgánico, principalmente desechos derivados de la 
preparación y consumo de alimentos, lo que equivale a unas 2611 t/día de residuos orgánicos (Bernache 2013).

El componente orgánico de los residuos está asociado a dos tipos de contaminación en los sitios de disposición final. El primero es la producción de gas metano, un gas tipo invernadero que se genera con la descomposición de la materia orgánica. El segundo, un aporte de humedad que contribuye de forma significativa a la producción de los llamados jugos de la basura, que se conocen como lixiviados. Tanto el metano como los lixiviados escapan de los sitios de disposición final, de manera que la materia orgánica en los residuos está asociada con la contaminación del aire por metano y la contaminación de fuentes de agua por lixiviados.

Una de las principales iniciativas para valorización de residuos orgánicos corresponde a la empresa Komposuiz, ubicada en el municipio de Zapopan. Dicha empresa inició sus actividades en 2009 y produce composta y mulch (cobertura orgánica que se extiende sobre suelos agrícolas o jardines) a partir del componente orgánico de los RSU, residuos orgánicos de agricultura y bagazo de agave de las tequileras de la región (Cobos 2015). De acuerdo con datos de la propia empresa, produce 10000 toneladas anuales de composta a partir de un monto original de 36000 toneladas de residuos orgánicos (Komposuiz 2017). En un estudio reciente, Cobos (2015) analizó los procesos de cinco plantas de producción de composta en México, Dicho estudio identifica a Komposuiz como la empresa con mejor desarrollo, comercialización y calidad de producto a nivel nacional. Este investigador señala que sólo Komposuiz entrega una ficha técnica con los datos del producto y la certificación de calidad por sus análisis de laboratorio (Cobos 2015).

Dicha operación sólo se lleva a cabo por Komposuiz, que comercializa el producto en costales y a granel... sus etiquetas sólo contienen las propiedades nutrimentales de la composta y una referencia general sobre los residuos que le dieron origen. Komposuiz presenta los datos de su producto de esa manera, debido a que su composta es producida bajo un proceso completamente normalizado y certificado, lo que le permite avalar que toda la composta que producen en su planta cumple con ciertos requisitos mínimos de calidad. Esto significa que, no importa el residuo del cual provenga la composta, el proceso normalizado asegura la calidad del producto terminado (Cobos 2015).
Esta es una de las acciones más importantes en el manejo de residuos orgánicos en la zona metropolitana de Guadalajara, en particular porque cumple con tres aportes a la sostenibilidad regional: evita que 36 mil t/año de residuos terminen enterrados en vertederos; disminuye la generación de gas metano en los sitios de disposición final, y aporta un abono orgánico que favorece la restauración de los suelos y reduce el uso de agroquímicos.

Otra acción importante para el aprovechamiento de residuos orgánicos es la que se lleva a cabo en el sitio de disposición final Relleno Sanitario Hasar's en el municipio de Zapopan, Jalisco. En dicho relleno se entierran RSU procedentes del municipio de Tlaquepaque, de recolección contratada y de empresas que llevan directamente sus residuos. En 2017 se depositaban en ese sitio un promedio de 1415 t/día de RSU, de las cuales 750 t correspondían a residuos orgánicos.

Desde 2008 iniciaron en Hasar's las actividades para la captura de gas metano. Este biogás es producido por bacterias que descomponen la materia orgánica de los RSU enterrados en contextos anaeróbicos. El procedimiento de preparación incluye la perforación de pozos de captación de gas; luego se colocan tapas, válvulas y mangueras para conducir el gas metano a la superficie y canalizarlo a un tanque de almacenamiento. La producción de metano se acelera cuando hay mayor humedad en el sustrato, por lo que los lixiviados son continuamente bombeados para irrigar la superficie del sitio con métodos de aspersión continua durante el día, lo cual satura de humedad el depósito de residuos.

El aprovechamiento del metano se puede realizar de dos formas: incineración en un horno controlado para generar dióxido de carbono, que es menos contaminante, o aprovechamiento para la generación de energía eléctrica. En el caso de Hasar's el procedimiento se enfoca a la incineración controlada, y los reportes indican que a principios de 2012 se incineraban $1300 \mathrm{~m}^{3} / \mathrm{h}$ (Rivera y Mendieta 2012).

El aprovechamiento de residuos orgánicos es de 99 t/día en la producción de composta, más las 750 t de orgánicos que se manejan en el Relleno Hasar's para generar metano; así, un total de 849 t/día del componente orgánico de los RSU son valorizadas en los cuatro municipios en estudio.

Sin embargo, el beneficio generado por el manejo que se realiza en Hasar's es parcial, ya que el procedimiento utilizado para aumentar la humedad en el sitio genera resultados contraproducentes. Por una parte, la humedad que se produce al irrigar con lixiviados la superficie del entierro acelera la pro- 
ducción de metano para obtener un flujo constante del gas, y la empresa obtiene un recurso económico por la venta de cada metro cúbico de gas capturado y sometido a incineración controlada (como parte del Protocolo de Kyoto). Así, la empresa obtiene una importante ganancia adicional por su sistema de manejo del gas, lo cual constituye su motivación principal; pero el riego constante de lixiviados por el método de aspersión genera una saturación de humedad en el sitio y produce escurrimientos de lixiviados hacia los taludes del entierro. El sitio no tiene una buena infraestructura para contener lixiviados, de tal manera que éstos regularmente escapan del sitio y llegan al cauce del Arroyo Grande de Milpillas, que corre pendiente abajo y genera degradación en el trayecto de $8 \mathrm{~km}$ hasta el río Santiago. Además, las actividades productivas de los residentes de tres poblaciones (Milpillas, Huaxtla y San Lorenzo) se han visto afectadas, ya que dependen en gran medida de la disponibilidad de agua suficiente y limpia para regadíos de huertas frutales y siembras de hortalizas. Desde 2009 los pobladores han presentado quejas y denuncias ambientales ante las instancias municipales y estatales correspondientes, con pocos resultados. El manejo irresponsable de lixiviados que tienen residuos peligrosos (plomo y cromo hexavalente) es la contraparte del método de saturar de humedad los residuos orgánicos para obtener mayor producción de gas metano.

\section{Reducción de los montos de RSU enterrados en sitios de disposición final}

El entierro de RSU ha sido y sigue siendo el principal método de disposición. En 1997 se enterraba el $95 \%$ de los residuos generados en los cuatro municipios de la ciudad, mientras que en 2017 este porcentaje ascendía al $92 \%$. En términos de porcentaje de los residuos enterrados, se tiene una disminución del $3 \%$ del total de RSU generados; sin embargo, esto ha sido un resultado del esfuerzo, en gran medida, del sector informal dedicado a la recolección - en sus diferentes modalidades- que realiza esa actividad por necesidad económica, más que por motivos ecológicos. El aprovechamiento de los RSU también ha sido resultado de iniciativas de asociaciones de colonos, centros escolares y universitarios, iglesias, organizaciones de la sociedad civil y otros grupos ciudadanos. En 2017, aún faltaban programas municipales de recolección de residuos separados en categorías primarias: orgánicos, inorgánicos y sanitarios. Ésta sería la base para desarrollar programas amplios de aprovechamiento y valorización de residuos.

El cuadro IV presenta los seis sitios de disposición final de RSU de los cuatro municipios que operaron durante el periodo de estudio. Los sitios de Matatlán en Tonalá y La Micaelita en Tlaquepaque ya no se encuentran en operación, mientras que los sitios de Picachos y Hasar's, que se ubican en el municipio de Zapopan, no habían entrado en operación en 1997.

Por una parte, se observa que la separación de residuos creció de 117 a 375 toneladas en el periodo 1997-2017. Por otra parte, en ese mismo periodo, los cuatro municipios pasaron de enterrar 3002 a 4690 t/día de RSU (Cuadro V), un incremento neto del $44 \%$.

Es importante señalar que ninguno de los seis sitios de disposición final identificados en el cuadro IV cumple cabalmente con las especificaciones de infraestructura, procesos de entierro, manejo del sitio

CUADRO V. SITIOS DE DISPOSICIÓN FINAL Y MONTOS DE RSU*

\begin{tabular}{llrr}
\hline Sitio de disposición final & $\begin{array}{l}\text { Municipios que } \\
\text { depositan en el sitio }\end{array}$ & 1997 & 2017 \\
\hline Matatlán & Guadalajara y Tonalá & 1142 & 0 \\
Laureles & Guadalajara y Tonalá & 517 & 2437 \\
El Taray & Zapopan & 1093 & 0 \\
Picachos & Zapopan & 0 & 1658 \\
La Micaelita & Tlaquepaque & 367 & 0 \\
Hasar's & Tlaquepaque & 0 & 833 \\
\hline Subtotal de ingresos al sitio & 3119 & 4927 \\
\hline \multicolumn{5}{c}{ Separación } & 117 & 237 \\
\hline Total de residuos enterrados & 2963 & 4690 \\
\hline
\end{tabular}

*En t/día 
y control de los vectores de contaminación (Bernache 2013, 2015). A pesar de que dos de los sitios cuentan con plantas para el tratamiento de lixiviados (Picachos y Hasar's), el manejo de éstos continúa siendo el principal problema por su contenido de residuos peligrosos (plomo y cromo hexavalente) que regularmente escapan del sitio en volúmenes importantes, en particular durante la temporada de lluvias, afectando los ecosistemas aledaños, las fuentes de agua (en particular el río Santiago y sus afluentes) y los sistemas productivos de las comunidades locales (Bernache 2015, Gran y Bernache 2016).

\section{DISCUSIÓN}

Los resultados indican que la producción de RSU se ha incrementado en la ZMG en un $58 \%$ durante el periodo de 20 años en estudio. La producción per cápita de RSU aumentó en $33 \%$ durante ese lapso. En términos de separación de residuos para reciclaje o valorización, creció $3 \%$ (del 5 al $8 \%$ del total de RSU).

La valorización de residuos orgánicos ha avanzado tanto en la producción de composta como en la generación de gas metano para su incineración controlada. Esto es positivo, aunque no es un reflejo de la participación social sino resultado de diversas motivaciones. En el caso del metano la motivación es puramente económica, lo ambiental es secundario y de hecho la irrigación de lixiviados aumenta el riesgo de derrames y escapes de este líquido contaminante al cauce del Arroyo Grande de Milpillas. En el caso de la empresa Komposuiz es un esfuerzo valioso en términos ambientales y también representa un negocio exitoso que va creciendo y sentando nuevos estándares para la industria de la composta.

Es importante señalar que cada ayuntamiento maneja los residuos de forma individual, es decir, no hay un proceso de integración y colaboración intermunicipal. Esto termina por afectar los sistemas de gestión integral, ya que cada ayuntamiento tiene su lógica y sus propios procesos. La intervención de compañías concesionarias en el manejo total o parcial de RSU no ha favorecido su gestión integral, ya que en la mayoría de los casos prevalece una visión empresarial de maximizar ganancias a costa de la degradación ambiental.

\section{CONCLUSIONES}

El manejo de residuos sólidos comprende cuatro actividades principales relacionadas con los servicios de recolección, transporte y transferencia, tratamiento y valorización, y disposición final. El título de este artículo hace referencia a dos nociones controversiales que han sido sustituidas por nuevos conceptos. Por una parte, las autoridades ya no enfatizan el manejo de residuos, sino la gestión y de preferencia la gestión integral sostenible. Por otra parte, se pide hablar de residuos sólidos y dejar atrás el concepto de basura. Esta propuesta de renovación parece tener cierto atractivo, ya que promueve el cambio y la mejora en este rubro importante de la dinámica urbana.

Sin embargo, el uso de nuevos conceptos no ha llevado a cambios significativos y, quizá, haya servido como una pantalla conceptual para simular que se está haciendo algo novedoso que se alinea con la visión del desarrollo sostenible, y que las acciones para evitar la degradación ambiental en este ámbito de los residuos han sido positivas. Lo cierto es que el uso de este nuevo vocabulario no ha podido transformar la realidad.

Los residuos siguen siendo basura, desechos inservibles que no son valorizados ni tratados, y su destino principal sigue siendo el tiradero, el vertedero y los llamados rellenos sanitarios (que tampoco cumplen cabalmente con la normatividad ambiental). Es decir, las políticas públicas y los programas de manejo no han ayudado a mejorar, de forma significativa, la gestión de los residuos, han propiciado una valorización parcial de cierto tipo de residuos que tienen valor en el mercado, pero en términos generales siguen ocasionando degradación ambiental regional por derrames de lixiviados peligrosos que afectan las fuentes de agua locales y regionales.

Por último, hablar de gestión es también una forma de implicar que se está trabajando de manera integral para que los residuos se separen, reciban algún tratamiento y se valoricen; que hay políticas públicas apropiadas y que se promueve la educación ambiental y la participación social. La realidad es que el manejo de la basura consiste en su recolección, transporte y entierro; lo demás no son más que programas esporádicos, con poca trascendencia y escaso impacto en la cultura del consumo. Este proceso de manejo es controlado, en la gran mayoría de los casos, de forma casi total por los gobiernos municipales que hacen lo que pueden con los recursos disponibles; sin embargo, en el caso de los cuatro municipios estudiados, los esfuerzos, iniciativas, programas y políticas públicas no han logrado convertir las prácticas de manejo mecánico de los residuos en una estrategia sólida de gestión sostenible de los RSU. 


\section{REFERENCIAS}

Aguayo N.G. (2013). Reciclaje de residuos en el área metropolitana de Guadalajara y su impacto en la sustentabilidad ambiental. Tesis de Maestría en Desarrollo Local y Territorio. Universidad de Guadalajara, Guadalajara, México, 223 pp.

Bernache G., Bazdresch M., Cuéllar J.L. y Moreno F. (1998). Basura y metrópoli. Gestión social y pública de los residuos sólidos municipales en la zona metropolitana de Guadalajara. Universidad de GuadalajaraCIESAS-ITESO-Colegio de Jalisco, Guadalajara, México, $238 \mathrm{pp}$.

Bernache G. (2013). Diagnóstico de residuos sólidos. Área Metropolitana de Guadalajara. Informe para ONU HABITAT Ciudades Prósperas (manuscrito inédito). Guadalajara, México, 12 pp.

Bernache G. (2015). La gestión de los residuos sólidos. Un reto para los gobiernos locales. Sociedad y Ambiente 7, 72-101.

Cobos V. (2015). Estudio sobre la normalización del proceso de compostaje. Tesis de Maestría en Administración. Unidad Profesional Interdisciplinaria de Ingeniería, Ciencias Sociales y Administrativas, Instituto Politécnico Nacional, Ciudad de México, 174 pp.

Gran J.A. y Bernache G. (2016). Gestión de residuos sólidos urbanos, capacidades del gobierno municipal y derechos ambientales. Sociedad y Ambiente 1 (9), 73-101.

IIEG (2017). Población y tasas de crecimiento promedio anual por sexo según municipio. Jalisco 1950-2015. Instituto de Información, Estadística y Geografía de Jalisco [en línea]. http://iieg.gob.mx/destino.php?l=\% 22contenido\%2FPoblacionVivienda\%2FPobVolumen ycrecimientomun 1950-2015.xlsx\%22\&s=297\&c=902

INEGI (2015). Cuéntame. Instituto Nacional de Estadística, Geografía e Informática, México [en línea]. http:// cuentame.inegi.org.mx/monografias/informacion/jal/ default.aspx?tema $=$ me\&e $=14$ 12/01/17

Komposuiz (2017). Komposuiz recreando vida [en línea]. http://komposuiz.com/ 12/01/17

SEMADES (2007). Ley de Gestión Integral de Residuos del Estado de Jalisco. Decreto 21798. Secretaría de
Medio Ambiente y Desarrollo Sustentable de JaliscoCongreso del Estado de Jalisco [en línea]. http://info. jalisco.gob.mx/sites/default/files/leyes/Ley_Gestion_Integral_Residuos_Jalisco.pdf 12/11/14

SEMADES (2008). NAE-SEMADES-007/2008. Acuerdo que establece los criterios y especificaciones técnicas bajo las cuales deberá realizar la separación, clasificación, recolección selectiva y valorización de los residuos en el Estado de Jalisco. Secretaría de Medio Ambiente y Desarrollo Sustentable de Jalisco. El Estado de Jalisco, periódico oficial, 16 de octubre [en línea]. http://info.jalisco.gob.mx/sites/default/files/ leyes/nae_residuos.pdf 19/10/14

SEMADES (2011). Reglamento de la Ley de Gestión Integral de los Residuos del Estado de Jalisco en materia de recolección y transporte de residuos de manejo especial. Secretaría de Medio Ambiente y Desarrollo Sustentable de Jalisco. El Estado de Jalisco, periódico oficial, 1 de julio [en línea]. http://transparencia.info. jalisco.gob.mx/sites/default/files/Reglamento $\% 20$ de\%20la\%20Ley\%20de\%20Gesti\%C3\%B3n\%20 Integral $\% 20 \mathrm{de} \% 201$ os $\% 20$ Residuos $\% 20 \mathrm{del} \% 20$ Estado\%20de\%20Jalisco.pdf 20/01/15

SEMARNAT (2003). Ley General para la Prevención y Gestión Integral de los Residuos. Secretaría de Medio Ambiente Recursos y Recursos Naturales, Cámara de Diputados, [en línea]. http://www.diputados.gob.mx/ LeyesBiblio/pdf/263_190118.pdf 14/11/14

SEMARNAT (2006). Reglamento de la Ley General para la Prevención y Gestión Integral de los Residuos. Secretaría de Medio Ambiente Recursos y Recursos Naturales, Cámara de Diputados [en línea]. http:// www.diputados.gob.mx/LeyesBiblio/regley/Reg_LGPGIR_311014.pdf. 20/01/15

Rivera E. y Mendieta E. (2012). Purifican sustancias tóxicas en un relleno sanitario de Jalisco. En; Gobernanza metropolitana [en línea]. https://gobernanzametropolitana.wordpress.com/tag/empresa-hasars/21/01/17

Vilches Larrea R.E. (2013). Testing benchmark indicators for integrated and sustainable waste and resource management. Tesis de Maestría en Ciencias de Ingeniería Ambiental. Imperial College, Londres, Reino Unido, $176 \mathrm{pp}$. 